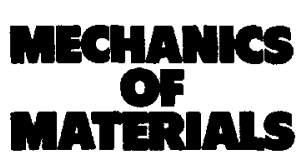

Mechanics of Materials 23 (1996) 217-228

\title{
Effective thermal conductivity of misoriented short-fiber reinforced thermoplastics
}

\author{
Chao-Hsun Chen ${ }^{*, 1}$, Yuh-Chung Wang ${ }^{2}$ \\ Institute of Applied Mechanics, National Taiwan University, 1. Sec, 4, Roosvelt Rd., Taipei, Taiwan
}

Received 7 September 1994; revised version received 25 July 1995; accepted 20 February 1996

\begin{abstract}
This paper deals with the effective thermal conductivity of composites containing misoriented fibers. Statistical distribution functions such as Weilbull can be used to represent this kind of distribution. The orientation distribution of fibers in FRTP (fiber-reinforced thermoplastics) can be characterized by a single parameter exponential function $F(\theta)=1-\mathrm{e}^{-\lambda \theta}$. A large $\lambda$ indicates a highly oriented material whereas a small $\lambda$ represents a quasi-isotropic material. A method based on the idea of Mori-Tanaka's mean field theory in conjunction with the Eshelby's equivalent inclusion method for steady-state heat conduction in composite including the effects of fiber length and orientation to predict thermal conductivity of FRTP is presented. The effective thermal conductivity of planar orientation distribution and transversely isotropic distribution of fibers are studied. For planar orientation case, we have examined the thermal conductivity constants of injection-molded tensile bars of poly (pheylene sulfide) reinforced with 30 and $40 \%$ by weight of carbon or glass fibers which have been measured by Choy et al. (1992). For transversely isotropic orientation case, the thermal conductivity constants of a transversely isotropic composite are explicitly derived. These thermal conductivity constants are given in terms of the length-diameter aspect ratio, the volume fraction, and the orientation-distribution parameter of the short fibers. Numerical results are presented to demonstrate the effects of the fiber thermal property, aspect ratio, volume fraction and orientation distribution function on composite thermal conductivity. The fiber orientation distribution and aspect ratio have a more significant effect on composite longitudinal thermal conductivity than does the fiber volume fraction within the range examined.
\end{abstract}

\section{Introduction}

Recently, thermoplastics reinforced by short glass or carbon fibers have received considerable attention, because they can be easily produced by the low-cost injection-molding process. Short fibers in these composites are misoriented due to the processing. De-

\footnotetext{
* Corresponding author.

1 Associate Professor.

${ }^{2}$ Graduate student.
}

spite the importance of this class of materials, there have been few detailed experimental and theoretical studies of the thermal conductivity, which are properties of great basic interest and technical significance.

To predict the thermal conductivity of a injection-molded fiber-reinforced thermoplastics, the simulated laminated-plate model is often used 1992. The degree of fiber orientation is expected to be higher in the skin layer, so to study this effect, measurements were made by Choy et al. (1992) in 
both the skin and core regions. They have measured thermal conductivities of injection-molded bars of PPS reinforced with 30 and $40 \mathrm{wt} \%$ of carbon or glass fibers. The distributions of the aspect ratio and orientation of the fibers were also determined. For short-fiber reinforced thermoplastics (FRTP), the two most important microstructural parameters are fiber length and fiber orientation.

A number of analytical models have been proposed to predict the effective thermal conductivity of a short fiber composite. These models however, were focused on either aligned shortfiber composite or completely random short fiber composite (Chou and Nomura, 1980-81). Recently Hatta and Taya (1985) proposed a new 'equivalent inclusion method' in the steady-state heat conduction by which the interaction among fibers at different orientations can be taken into account.

An orientation distribution generally requires a three dimensional description. However, if a specimen has a geometry such that the fiber distribution function is axisymmetric with respect to the major axis of the specimen, say 1-axis, so that the material as a whole posses a transversely isotropic in the 2-3 plane. Kacir et al. (1975a); Kacir et al. (1975b); Kacir et al. (1978) proposed a distribution function representing this planar orientation which has the property such that variation of its function's shape is able to describe a change from a unidirectional distribution to a random planar distribution.

In this paper we consider the effect of the distribution in fiber orientation on the effective thermal conductivity. Similar to the equivalent inclusion method of Hatta and Taya (1985), the interaction among fibers at different orientations is included in the analysis by adopting the mean stress concept of Mori and Tanaka (1973) and put it together with the eigen-strain idea of Eshelby (1957) to predict the effective thermal conductivity of FRTP. The orientation distribution of fibers in composite is characterized by Kacir's single parameter exponential function. These thermal conductivities are given in terms of the length-diameter aspect ratio, the volume fraction, and the orientation-distribution parameter of the short fibers. These effective thermal conductivities are given in very simple, explicit forms. Numerical results are presented to demonstrate the effects of fiber elastic property, aspect ratio, volume fraction and orientation distribution function on composite thermal conductivity.

In order to compare the theoretical model and the experiment, we introduce the experimental data measured by Choy et al. (1992). The orthotropy of the injection-molded thermoplastics (FRTP) is due to the fact that the fiber length distribution in FRTP has an asymmetric character with a tail at the long fiber ends.

\section{Effective thermal conductivity}

Now consider a representative volume of a fiberreinforced composite which is assumed to be statistically homogeneous. If an infinite homogeneous body (without inclusions, i.e., the matrix only) is subjected to a uniform heat flux $q_{0}$. The uniform temperature gradient $\nabla T_{\mathrm{o}}$ produced is

$\nabla T_{0}=K_{\mathrm{m}}^{-1} \cdot q_{0}=H_{0}$,

in which $K_{\mathrm{m}}$ is the thermal conductivity tensor of the matrix (which is assumed to be isotropic) and $H_{0}$ is the temperature gradient (i.e, $H_{0}=\nabla T_{0}$ ) of the matrix. When there are ellipsoidal inclusions (fibers) present in the matrix, a perturbed heat flux is induced and is denoted as $\tilde{q}(x)$. The total heat flux $q(x)$ is now the sum of two heat flux $q_{0}+\tilde{q}(x)$. Define the volumetric average of the perturbed heat flux and temperature gradient of the matrix, $\bar{q}$ and $\bar{\nabla} T$ according to

$$
\begin{aligned}
\bar{q} & =\langle\tilde{q}(x)\rangle_{D-\Omega} \\
& =\frac{1}{V_{D-\Omega}} \int_{D-\Omega} \tilde{q}(x) \mathrm{d} V=K_{\mathrm{m}} \nabla T=K_{\mathrm{m}} \bar{H},
\end{aligned}
$$

in which $D, \Omega, D-\Omega$ are the domain of the whole elastic body (composite), all the fibers and the matrix respectively, $V_{D-\Omega}$ is the volume of the matrix, \langle\rangle$_{D-\Omega}$ is the volumetric average for the domain $D-\Omega$, the superscript '-' means the volumetric average perturbed term for the domain $D-\Omega$. Thus the average heat flux in the matrix is

$q_{\mathrm{m}}=q_{0}+\bar{q}=K_{\mathrm{m}}\left(H_{0}+\bar{H}\right)$.

If a composite containing a single phase of ellipsoidal inhomogeneities. When all inhomogeneities are aligned in one direction, the following two equation (Taya and Mura, 1981) are obtained. 
$K_{\mathrm{m}}\left[\bar{H}+f_{1}\left(H^{\mathrm{pt}}-H^{*}\right)\right]=0$.

Denote $K_{\mathrm{f}}$ as the thermal conductivity of the fiber.

$$
\begin{aligned}
q_{\mathrm{f}} & =K_{\mathrm{f}} \cdot \nabla T_{\mathrm{f}}=K_{\mathrm{f}} \cdot H_{\mathrm{f}}=K_{\mathrm{f}} \cdot\left(H_{0}+\bar{H}+H^{\mathrm{pt}}\right) \\
& =K_{\mathrm{m}} \cdot\left(H_{0}+\bar{H}+H^{\mathrm{pt}}-H^{*}\right),
\end{aligned}
$$

where $H^{*}$ is the Eshelby's (1957) equivalent transformation temperature gradient (or Mura's eigenstrain, 1987). The perturbed temperature gradient $H^{\mathrm{pt}}$ is taken to be related to $H^{*}$ as

$H^{\mathrm{pt}}=\mathrm{S} \cdot H^{*}$

in which $\mathbf{S}$ is the Eshelby tensor associated with spheroidal inclusions (Eshelby, 1957; Mura, 1982). The nonzero components of Eshelby's tensor $\mathbf{S}$ are included in the Appendix.

Furthermore, the total volumetric average temperature gradient of the composite material $H^{\mathrm{T}}$ can be represented as

$H^{\mathrm{T}}=f_{0}\left(H_{0}+\bar{H}\right)+f_{1}\left(H_{0}+\bar{H}+H^{\mathrm{pt}}\right)=H_{0}+f_{1} H^{*}$

The result of Eq. (2.7) is derived by using (2.4). From Eqs. (2.5) and (2.6) we have

$H^{*}=\mathbf{A} \cdot \mathbf{T} \cdot\left(H_{0}+\bar{H}\right)=\mathbf{B} \cdot\left(H_{0}+\bar{H}\right)$,

in which $\mathbf{A}, \mathbf{T}$ and $\mathbf{B}$ can be represented as

$\mathbf{B}=\mathbf{A} \cdot \mathbf{T}$,

$\mathrm{A}=\mathrm{I}-K_{\mathrm{m}}^{-1} \cdot K_{\mathrm{f}}$,

$\mathbf{T}=\left(\mathbf{I}+\mathbf{S} \cdot K_{\mathrm{m}}^{-1} \cdot K_{\mathrm{f}}-\mathbf{S}\right)^{-1}$

and $I$ is the fourth order tensor which can be defined as

$\mathbf{I}_{p q}=\left\{\begin{array}{lll}1 & \text { for } & p==q \\ 0 & \text { for } & p \neq \neq\end{array}\right.$.

Now from (2.6) we have

$$
\begin{aligned}
H^{\mathrm{pt}}-H^{*} & =(\mathbf{S}-\mathbf{I}) \cdot \mathbf{A} \cdot \mathbf{T} \cdot\left(H_{0}+\bar{H}\right) \\
& =\mathbf{E} \cdot\left(H_{0}+\bar{H}\right),
\end{aligned}
$$

where

$\mathbf{E}=(\mathbf{S}-\mathbf{I}) \cdot \mathbf{A} \cdot \mathbf{T}$.

Substituting Eq. (2.11) into Eq. (2.4) leads to

$\bar{H}+f_{1} \mathbf{E} \cdot\left(H_{0}+\bar{H}\right)=0$.
Rearranging Eq. (2.13) we have

$H_{0}+\bar{H}=\left(I+f_{1} \mathbf{E}\right)^{-1} \cdot H_{0}$.

Substituting (2.14) into (2.7) leads to

$H^{\mathrm{T}}=\left[\mathbf{I}+f_{1} \mathbf{B} \cdot\left(\mathbf{I}+f_{1} \mathbf{E}\right)^{-1}\right] \cdot H_{0}$.

The effective thermal conductivity can be defined based on the following relation

$q_{0}=K^{*} \cdot H^{\mathrm{T}}$.

Therefore, the effective thermal conductivity then can be determined from Eqs. (2.1), (2.16) and (2.17) as follows:

$K^{*}=K_{\mathrm{m}}\left[\mathbf{I}+f_{1} \mathbf{B} \cdot\left(I+f_{1} \mathbf{E}\right)^{-1}\right]^{-1}$.

Consider the distribution of fiber orientation. The coordinate system is defined so that the fibers are in the 1-2 plane with the orientation angle $\theta$ being measured relative to the 1 -axis. Let $x_{i}^{\prime}$ be the local coordinate system of the inclusion, and $x_{i}$ be the global coordinate system of whole elastic body (composite material). By using the spherical coordinate system, their relation then can be represented as $x^{\prime}=Q \cdot x$

where

$Q_{i j}=\left[\begin{array}{ccc}\cos \theta & \sin \theta \cos \phi & \sin \theta \sin \phi \\ -\sin \theta & \cos \theta \cos \phi & \cos \theta \sin \phi \\ 0 & -\sin \phi & \cos \phi\end{array}\right]$.

Since $\mathbf{B}$ and $\mathbf{E}$ in Eq. (2.17) are both second order tensor, after being transformed into the global coordinate system then can be written as a representative form

$A_{i j}^{\prime}=Q_{i p} Q_{j q} A_{p q}$

The general expression of the volumetric average after arbitrary material forming history becomes

$\langle\mathbf{A}\rangle=\overline{A_{i j}}=\frac{1}{V} \int_{-\phi^{\prime}}^{\phi^{\prime}} \int_{-\theta^{\prime}}^{\theta^{\prime}} A_{i j}^{\prime} f(\theta) \mathrm{d} \theta \mathrm{d} \phi$,

where $\phi^{\prime}$ and $\theta^{\prime}$ are the ranges of integration in the $\phi$ and $\theta$ direction, respectively, and $V$ is the volume of relevant segment of a sphere with unit radius.

Therefore, the general expression for the effective 
thermal conductivities, $K^{*}$, after any material forming process described above can be calculated by assuming statistically homogeneity and integrating the thermal conductivities determined by substituting the relation of Eq. (2.21) into Eq. (2.17) as follows:

$K^{*}=K_{\mathrm{m}}\left[\mathbf{I}+f_{1} \overline{\mathbf{B}} \cdot\left(I+f_{1} \overline{\mathbf{E}}\right)^{-1}\right]^{-1}$

where

$\overline{\mathbf{B}}=\langle\mathbf{B}\rangle$ and $\overline{\mathbf{E}}=\langle\mathbf{E}\rangle$.

\section{Fiber-orientation distribution}

Any material forming processes, such as injection, extrusion and rolling of the initially isotropic material, causes a permanent fiber orientation which changes the material properties from isotropic to either transversely isotropic or orthotopic. The changes are related in a complex way to the geometry of the fibers, melt behavior of the polymer matrix, mold cavity and the filling process. An orientation generally requires a three dimensional description.

If a symmetrical angular fiber distribution function around the major alignment direction is assumed. Thus the experimental fiber distribution data can be used to build a complex symmetrical laminate of weighted groups of angle plies oriented $\pm \theta^{\circ}$ from the major alignment axis. With this assumption, Kacir et al. (1975a); Kacir et al. (1975b), proposed a single parameter exponential function to describe the fiber orientation distribution in a molded specimen. Definition of Kacir's orientation distribution function is as follows:

$\rho(\theta)=\lambda \mathrm{e}^{-\lambda \theta}$

$F(\theta)=\int_{0}^{\theta} \lambda \mathrm{e}^{-\lambda x} \mathrm{~d} x=1-\mathrm{e}^{-\lambda \theta}$,

where $F(\theta)$ is the accumulated percent of fiber oriented between 0 to $\pm \theta, \rho(\theta)$ is the density function of fibers oriented at $\pm \theta ; \lambda$; is the shape parameter, and $\theta$ is the orientation angle ranging from 0 to $\pi / 2$. Large $\lambda$ indicates that fibers have a major preferential alignment. As $\lambda$ decreases, the orientation of fibers in the sample will become more random.

\subsection{Planar orientation of fibers}

However, if a thin sheet specimen has a geometry such that the thickness dimension is much less than the width and length dimensions, then a simplified planar orientation of fibers can be assumed. Thus Eq. (2.21) can be defined as follows:

$$
\begin{aligned}
& \bar{A}=\left\langle\underset{\sim}{A}\left(\theta, \phi_{0}\right)\right\rangle \\
& \equiv\left[\int_{-\pi / 2}^{0} A^{\prime}\left(\theta, \phi_{0}\right) \rho(-\theta) \mathrm{d} \theta\right. \\
& \left.+\int_{0}^{\pi / 2} A^{\prime}\left(\theta, \phi_{0}\right) \rho(\theta) \mathrm{d} \theta\right] \\
& \times\left[2 \int_{0}^{\pi / 2} \rho(\theta) d \theta\right]^{-1}
\end{aligned}
$$

\subsection{Transverse isotropic in the 2-3 plane}

In this section we consider the case where the distribution function $\rho(\theta, \phi)$ is axisymmetric with respect to the major axis of the specimen, say $x_{1}$ axis, so that the material as a whole possesses a transversely isotropic properties in the $2-3$ plane. It means that $\rho$ is function of $\theta$ only. Thus, Eq. (2.21) can be defined as follows:

$$
\begin{aligned}
\underset{A}{A}= & \langle\underset{\sim}{A(\theta, \phi)\rangle} \\
\equiv & {\left[\int_{0}^{2 \pi} \int_{0}^{\pi / 2} A^{\prime}(\theta, \phi) \rho(\theta) \sin \theta \mathrm{d} \theta \mathrm{d} \phi\right] } \\
& \times\left[\int_{0}^{2 \pi} \int_{0}^{\pi / 2} \rho(\theta) \sin \theta \mathrm{d} \theta \mathrm{d} \phi\right]^{-1} .
\end{aligned}
$$

\section{Results and discussion}

\subsection{Thermal conductivity of the matrix and fibers}

The thermal conductivity of a short fiber-reinforced composite depend on the thermal conductivity of the fiber and matrix, the volume fraction $f_{1}$ aspect ratio, $l / d$, and the degree of orientation (characterized by the parameter $\lambda$ ) of the fibers. Their values are shown in Table 1 (from Choy et al., 1992). 
Table 1

The thermal conductivity and density of carbon and glass fibers and PPS matrix

\begin{tabular}{lccl}
\hline & $\begin{array}{l}\text { Carbon fiber } \\
\text { (Thronel 300) }\end{array}$ & $\begin{array}{l}\text { E glass } \\
\text { fiber }\end{array}$ & $\begin{array}{l}\text { PPS } \\
\text { matrix }\end{array}$ \\
\hline$K_{11}(\mathrm{~mW} / \mathrm{cm} \mathrm{K})$ & 94 & 10.4 & 2 \\
$K_{22}(\mathrm{~mW} / \mathrm{cm} \mathrm{K})$ & 6.7 & 10.4 & 2 \\
$v_{12}$ & 0.2 & 0.25 & 0.4 \\
$v_{23}$ & 0.25 & 0.25 & 0.4 \\
$\rho\left(\mathrm{g} / \mathrm{cm}^{3}\right)$ & 1.75 & 2.56 & 1.36 \\
\hline
\end{tabular}

The fibers have a much higher thermal conductivity than the matrix, and this would lead to thermal conductivity enhancement in composites. The effect is expected to be much stronger in carbon-fiber-reinforced composites since $K_{\mathrm{f}}^{11} / K_{\mathrm{m}}^{11}=47$ for carbon fibers compared with 5.2 for glass fibers.

\subsection{Thermal conductivity of injection-molded short- fiber reinforced thernoplastics}

\subsubsection{Fiber orientation and aspect ratio}

In the foregoing Section 3.2, if a thin sheet specimen has a geometry such that the thickness dimension is much less than the width and length dimensions, then a simplified planar orientation of fiber can be assumed.

We have employed the experimental results of Choy et al. (1992), for the orientation of fibers in the surface layer (at a depth of $0.3 \mathrm{~mm}$ ) and middle layer (at a depth of $1.6 \mathrm{~mm}$ ) of three composites, PPS30cf, PPS40cf, and PPS40gf, as follows:

(1) The fibers in the surface layer are aligned close to the mold flow direction (1'-axis), making an average angle $\langle\theta\rangle$ of about $15^{\circ}$ with this axis. More-
Table 2

The volume fraction, $f_{1}$, averaged aspect ratio, $l / d$, and orientation factor, $\lambda$ of the fibers in three composites (Choy et al., 1992)

\begin{tabular}{lllll}
\hline & $f_{1}$ & Average aspect ratio & & \\
\cline { 3 - 5 } & & & surface layer & middle layer \\
\hline PPS30cf & 0.24 & 21 & 4.1 & 1.9 \\
PPS40cf & 0.34 & 16 & 4.7 & 2.1 \\
PPS40gf & 0.26 & 17 & 5.3 & 2.9 \\
\hline
\end{tabular}

over, the fiber orientation is roughly the same at all positions along the width.

(2) The fiber orientation is also very high near the two edges (width positions 0.5 and $12 \mathrm{~mm}$ ) of the middle layer, but becomes increasingly random as one moves toward the core (width position 4-8 $\mathrm{mm}$ ).

(3) The fiber orientation distribution for a PPS30cf sample trimmed to a width of $8 \mathrm{~mm} \mathrm{(} \pm 4 \mathrm{~mm}$ from central line) showed that more than $60 \%$ of the fibers in the surface layer lie at an angle of less than $15^{\circ}$ relative to the $1^{\prime}$-axis, and there are almost no fibers in the range of $60^{\circ}$ to $90^{\circ}$. The middle layer exhibits a broader angular distribution, with a significant number of fibers lying at large angles.

(4) The fibers in each composite have a wide range of aspect ratios.

However, the average aspect ratio falls within the narrow range of 16-21 for all three composites. From these data the cumulative fiber distribution $F(\theta)$, ie., the fraction of fibers oriented at an angles smaller than $\theta$, was calculated and by fitting the $F(\theta)$ data to the expression in Eq. (3.2), the orientation factors $\lambda$ for the three composites were obtained as Table 2. It should be noted that $\lambda=0$ and $\lambda=\infty$ correspond to random and perfect orientation, respec-

Table 3

The thermal conductivity (in unit of $\mathrm{mW} / \mathrm{cm} \mathrm{K}$ ) of three composites

\begin{tabular}{|c|c|c|c|c|c|c|}
\hline & \multicolumn{2}{|c|}{ PPS30ct $(24.3 \%)$} & \multicolumn{2}{|c|}{ PPS40cf(33.5\%) } & \multicolumn{2}{|c|}{ PPS40gf(26.4\%) } \\
\hline & surface & middle & surface & middle & surface & middle \\
\hline$\lambda$ & 4.1 & 1.9 & 4.7 & 2.1 & 5.3 & 2.9 \\
\hline$K_{11}$ & $\begin{array}{c}15.8 \\
(15.2)\end{array}$ & $\begin{array}{c}13.8 \\
(12.4)\end{array}$ & $\begin{array}{c}19.1 \\
(17.2)\end{array}$ & $\begin{array}{c}16.5 \\
(15.6)\end{array}$ & $\begin{array}{c}4.10 \\
(4.08)\end{array}$ & $\begin{array}{c}4.03 \\
(3.99)\end{array}$ \\
\hline$K_{22}$ & $\begin{array}{c}3.33 \\
(4.31)\end{array}$ & $\begin{array}{c}5.15 \\
(5.50)\end{array}$ & $\begin{array}{c}3.52 \\
(4.69)\end{array}$ & $\begin{array}{c}5.67 \\
(5.70)\end{array}$ & $\begin{array}{c}2.70 \\
(3.24)\end{array}$ & $\begin{array}{c}2.80 \\
(3.22)\end{array}$ \\
\hline
\end{tabular}

The values between parentheses are the experimental results of Choy et al. (1992). 
tively, and so the value of $\lambda$ provides a quantitative measure of the degree of fiber alignment. All three composites have similar $\lambda$ values, with $\lambda=4.1-5.3$ in the surface layer and 1.9-2.9 in the middle layer.

\subsubsection{Thermal conductivity of the composites}

The thermal conductivity, $K^{*}$, of the composites are given in Table 3. Compared with the thermal conductivity of $K_{\mathrm{m}}^{11}=2 \mathrm{~mW} / \mathrm{cm} \mathrm{K}$ for the PPS matrix, the thermal conductivity of the composites are significantly higher, clearly revealing the en- hancement effect of the fibers. Since the surface layer has a higher degree of fiber orientation than the middle layer, it has a higher $K_{11}$ but lower $K_{22}$ (skin-core effect). The alignment of fibers also leads to a strong anisotropy in the elastic properties. $K_{11}$ for carbon fiber composites is substantially higher than $K_{22}$. These anisotropies reflect the high anisotropy of the fiber. Since glass fiber is isotropic and has a lower thermal conductivity than carbon fiber, $K_{11}$ for the glass fiber composite at a similar $f_{1}$ is lower than that of carbon fiber composite by a factor of about 4 (the $K_{11}$ values of $\operatorname{PPS} 30 \mathrm{cf}\left(f_{1}=\right.$
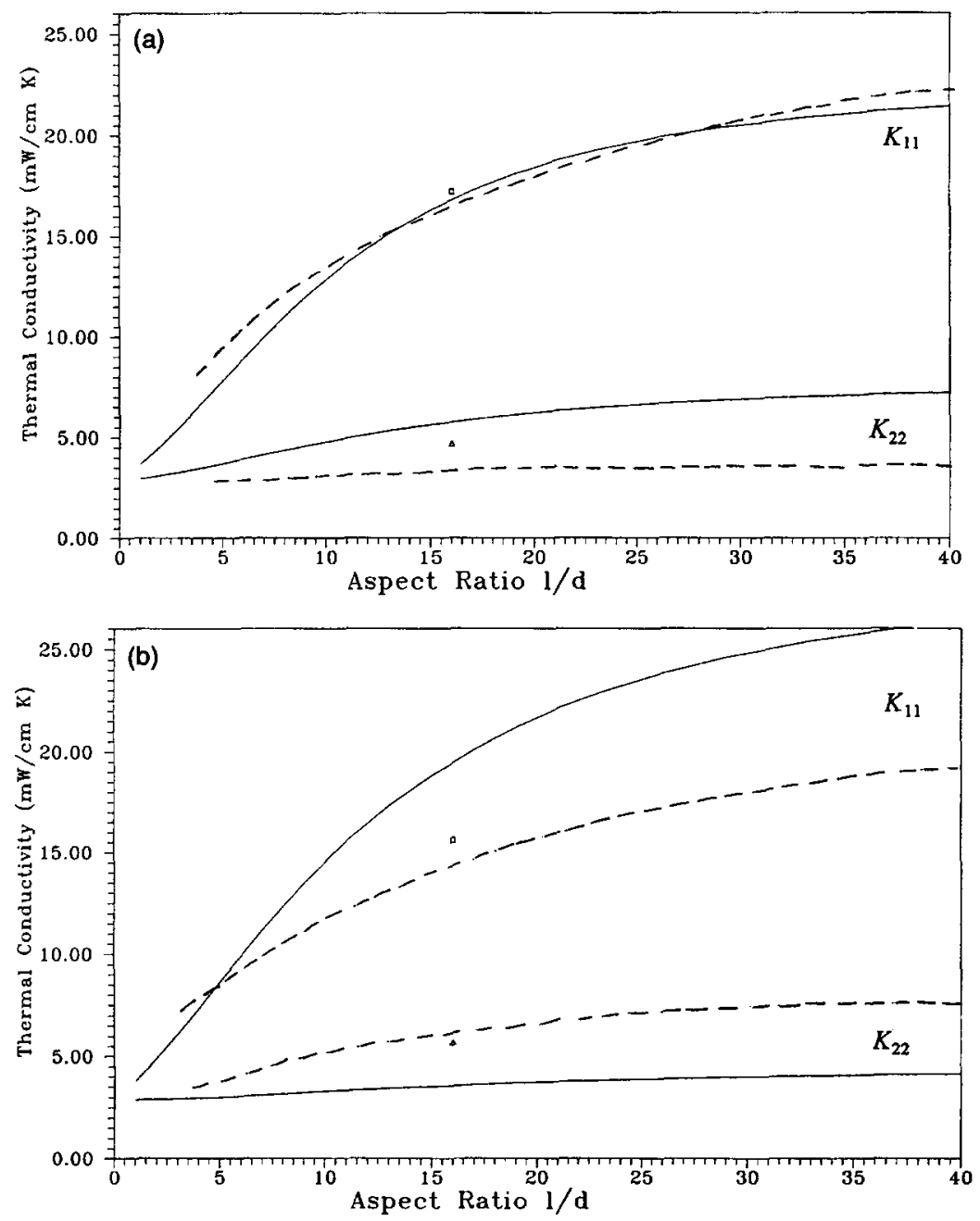

Fig. 1. Thermal conductivity of $40 \mathrm{wt} \%$ carbon-fiber-reinforced PPS as functions of fiber aspect ratio: (a) surface layer, (b) middle layer; symbols represent the experimental data. The theoretical curves are calculated by taking $\lambda=4.7$ and 2.1 in (a) and (b), respectively. (Solid line-present model; dashed line-laminated plate model.) 
$0.24)$ and PPS40gf $\left(f_{1}=0.26\right)$ in Table 3 may be compared).

Besides fiber orientation, there are two other factors that influence the thermal conductivity: fiber volume fraction $f_{1}$, and aspect ratio $l / d$. Table 3 shows that for glass fiber composite, $K_{11}$ is roughly proportional to $f_{3}$, but the dependence is weaker for others. However, $K_{11}$ for PPS $40 \mathrm{cf}$ is only $10 \%$ higher than that for PPS30cf although $f_{1}$ is more than $30 \%$ higher. The small difference in $K_{11}$ arises largely from the fact that PPS40cf has a much smaller aspect ratio (see Table 2). For the three composites samples, PPS30cf, PPS40cf and PPS40gf, all the relevant parameters have been determined, and the thermal conductivity can be calculated according to the Mori-Tanaka mean field theory in conjunction with Eshelby's equivalent-inclusion method discussed earlier (see Theory). As shown in
Table 4

Comparison of the experimental and theoretical values of the thermal conductivity (in unit $\mathrm{mW} / \mathrm{cm} \mathrm{K}$ ) of three composites

\begin{tabular}{llrrr}
\hline & \multicolumn{4}{c}{ Deviation (\%) } \\
\cline { 3 - 5 } & & PPS30cf & PPS40cf & PPS40gf \\
\hline Surface layer & $K_{11}$ & -4 & -11 & -1 \\
& $K_{22}$ & 23 & 25 & 17 \\
Middle layer & $K_{11}$ & -11 & -6 & -1 \\
& $K_{11}$ & 6 & 1 & 13 \\
\hline
\end{tabular}

Table 4 the theoretical predictions and experimental results differ by less than $20 \%$ on the average.

The good agreement implies that the structural model we have used to describe injection-molded composites is reasonable and that the theory provides a satisfactory theoretical framework.

To gain a deeper understanding of the effects of various parameters, we have calculated and plotted
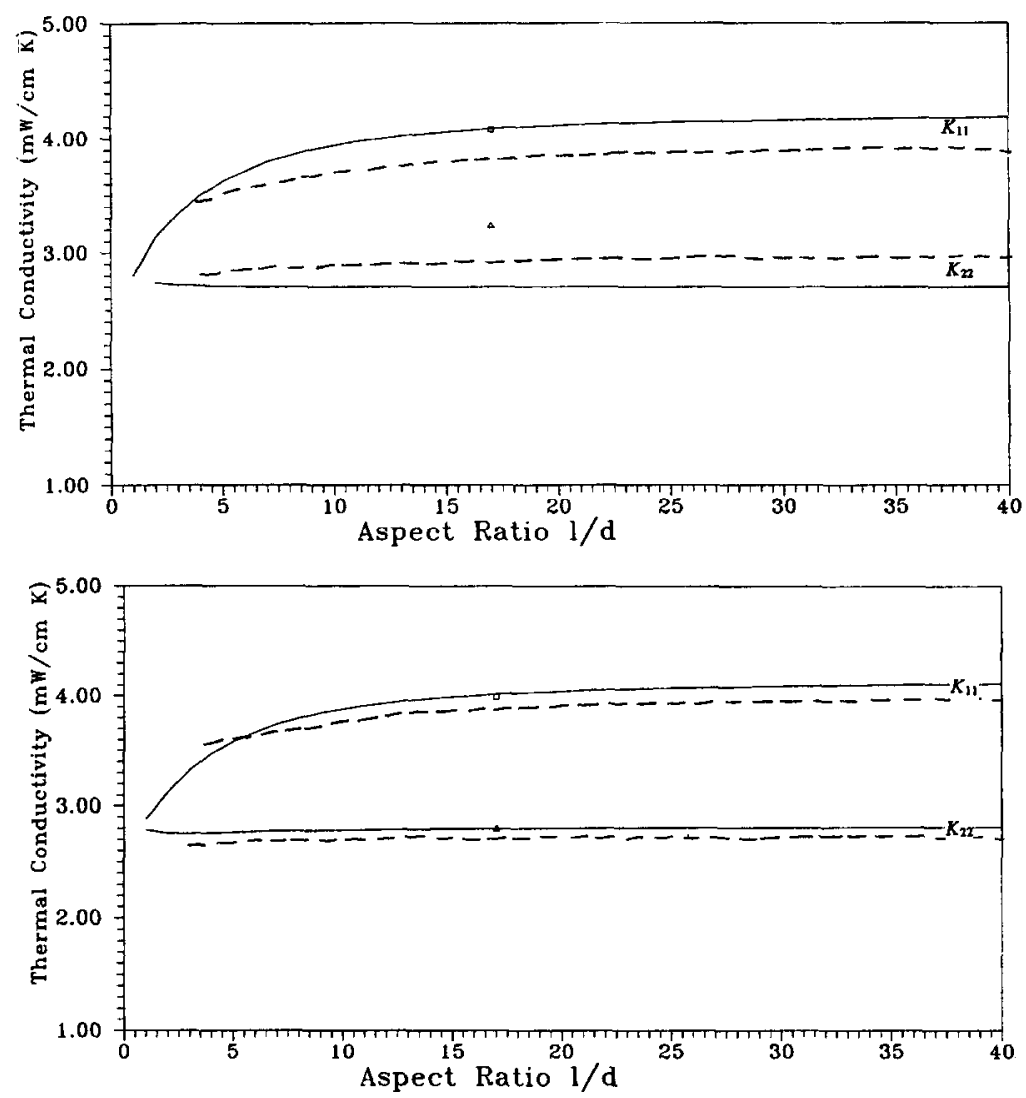

Fig. 2. Thermal conductivity of 40 wt\% glass-fiber-reinforced PPS as functions of fiber aspect ratio: (a) surface layer, (b) middle layer; symbols represent the experimental data. The theoretical curves are calculated by taking $\lambda=5.3$ and 2.9 in (a) and (b), respectively. (Solid line-present model; dashed line-laminated plate model.) 
the elastic moduli as functions of the aspect ratio, $l / d$. Fig. 1 shows that all the thermal conductivity for carbon fiber composites increase with increasing $l / d$, but the increase is much more significant for the longitudinal thermal conductivity $K_{11}$. In the $l / d$ range of 10-20, $K_{11}$ for both layers increase almost linearly with $l / d$. These results emphasize the importance of choosing suitable processing technique to increase the fiber aspect ratio. The situation is somewhat different for glass-fiber-reinforced composites (Fig. 2). Although $K_{11}$ also increases sharply at low aspect ratio, the effect becomes saturated above $l / d=15$. This arises from the fact that $K_{11}$ of glass fibers is much smaller than that of carbon fibers.

Next we turn our attention to the dependence on fiber volume fraction $f_{1}$ (Figs. 3 and 4). In the typical $f_{1}$ range of $0.2-0.35$ for short-carbon-fiber composites, $K_{11}$ increases slightly faster than linearly with $f_{1}$ at all fiber orientations. However, the slope of the curve becomes smaller than as $\lambda$ decreases. At $f_{1}=0.335$ (the fiber volume fraction of PPS40cf), $K_{11}$ is higher than the matrix modulus $K_{\mathrm{m}}^{11}$ by a factor of 4.5 for fibers randomly oriented in the $1^{\prime}-2^{\prime}$ plane $(\lambda=0)$ and 1 for fibers aligned in the $1^{\prime}$ direction. $(\lambda=\infty)$. This is the range of enhancement we can expect for the thermal conductivity along the flow direction of injection-molded bars. The orientation factor $(\lambda=4.7)$ of the surface layer of PPS $40 \mathrm{cf}$ is sufficiently high that its $K_{\mathrm{f}}^{11} / K_{\mathrm{m}}^{11}$ value of 8.6 .

It is now of interest to see how the fiber distribution - (i) when it changes from uniaxial directional $(\lambda=\infty)$ to random distribution $(\lambda=0)$, (ii) the volume fraction, $f_{1}$, (iii) the aspect ratio, $l / d$, of the reinforced fibers - would effect the thermal conductivity of a orthotopic composite. We have calculated and plotted the fiberorientation dependence the thermal conductivity of $f_{1}=30 \%$ glass-fiber-reinforced PPS as functions of the aspect ratio, $l / d$, in Fig. 3a and $b$. They show that the thermal conductivity $K_{11}, K_{22}$ increase with increasing $l / d$. The effect becomes saturated above $l / d=50$.

Next we turn our attention to the fiber volume fraction, $f_{1}$, dependence of thermal conductivity as function of $\lambda$. The values of $f_{1}=0.1,0.2,0.3$ and 0.4 were chosen for demonstrations. The thermal conductivity of the composite as function of $\lambda$ are
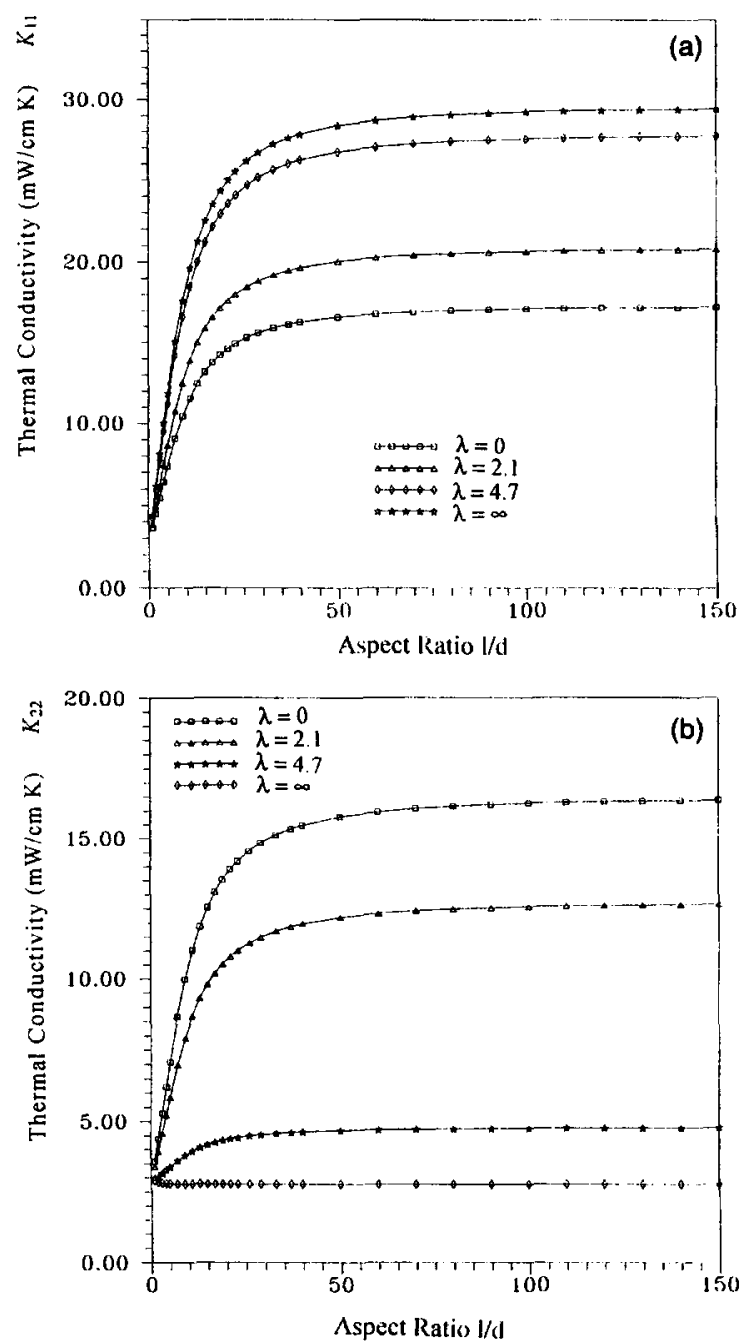

Fig. 3. The fiber orientation factor $\lambda$ dependence of the thermal conductivity as functions of aspect ratio, $l / d$, at $f_{1}=30 \%$; (a) $K_{11}$, (b) $K_{22}$.

decipted in Fig. 4a and b. The thermal conductivity $K_{11}$ increase with increasing $\lambda$ but for $K_{22}$ decrease with increasing $\lambda$. The effect becomes saturated above $\lambda=10$.

\subsection{Thermal conductivity of transversely isotropic short-fiber reinforced thermoplastics}

\subsubsection{Explicit expression of the thermal conductivity}

In this section, we will derive the explicit form of the thermal conductivity. Following the foregoing process the effective thermal conductivity $K^{*}$ in Eq. 

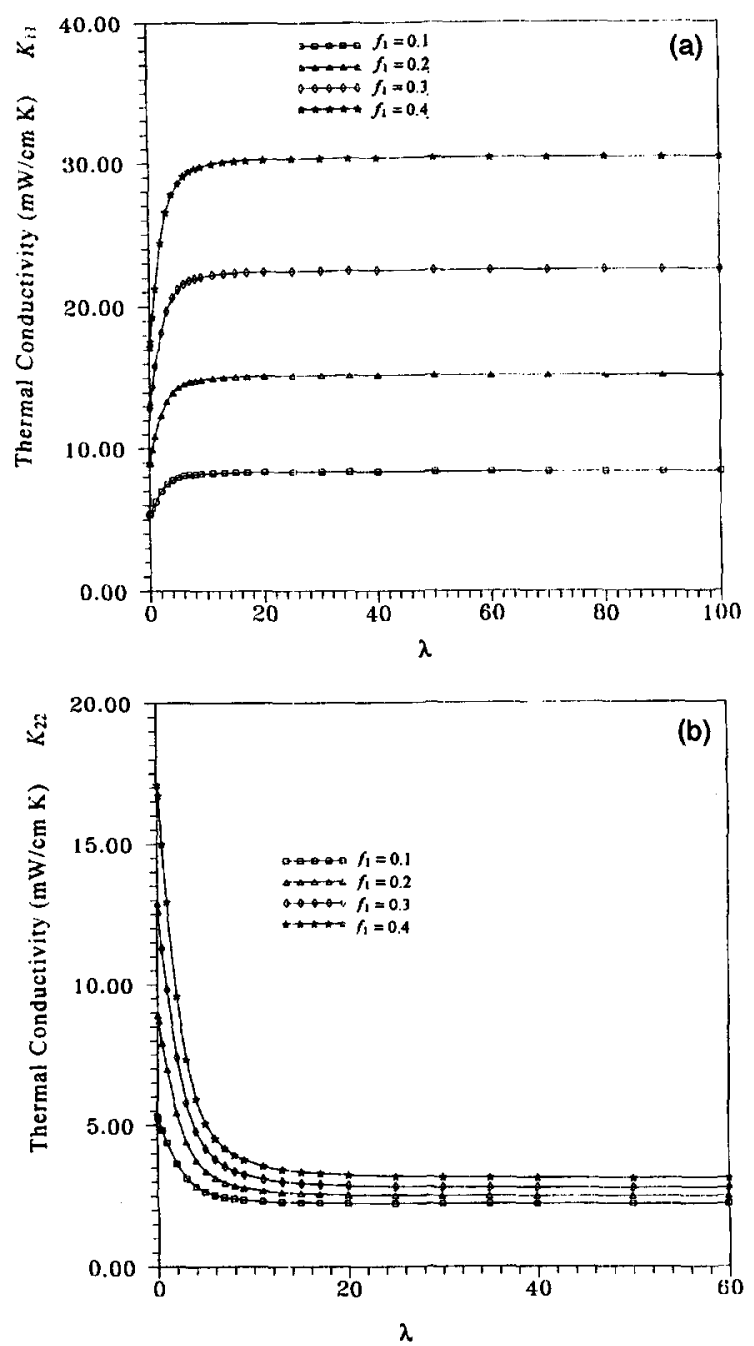

Fig. 4. The volume fractions dependence of the thermal conductivity as functions of fiber orientation factor, $\lambda$, at $l / d=15$; (a) $K_{11}$, (b) $K_{22}$.

(2.22). Then the thermal conductivity of a transversely isotropic composite can be explicitly expressed as follows:

$K^{*}=\left[\begin{array}{ccc}K_{11}^{*} & 0 & 0 \\ 0 & K_{22}^{*} & 0 \\ 0 & 0 & K_{22}^{*}\end{array}\right]$, where

$$
K_{11}^{*}=K_{\mathrm{m}}^{11} \frac{1+f_{1} \bar{E}_{11}}{1+f_{1} \bar{E}_{11}+f_{1} \bar{B}_{11}}
$$

and

$$
K_{22}^{*}=K_{m}^{22} \frac{1+f_{1} \bar{E}_{22}}{1+f_{1} \bar{E}_{22}+f_{1} \bar{B}_{22}} .
$$

Since $\bar{B}$ and $\bar{E}$ has the similar form, we can use $\bar{A}$ to represent $\bar{B}$ and $\bar{E}$. And its nonzero components can be written as follows:

$$
\begin{aligned}
\bar{A}_{11}= & \frac{1}{4}\left(A_{11}^{\prime}+3 A_{22}^{\prime}\right) b_{1}-\frac{1}{4}\left(A_{11}^{\prime}+3 A_{22}^{\prime}\right) b_{2} \\
& +\frac{3}{4}\left(A_{11}^{\prime}-A_{22}^{\prime}\right) b_{3}+\frac{1}{4}\left(A_{11}^{\prime}-A_{22}^{\prime}\right) b_{4} \\
& -2\left(A_{12}^{\prime}+A_{21}^{\prime}\right) b_{5}-\left(A_{12}^{\prime}+A_{21}^{\prime}\right) b_{6}, \\
\bar{A}_{12}= & 0, \quad \bar{A}_{13}=\bar{A}_{12}, \quad \bar{A}_{21}=\bar{A}_{12}, \\
\overline{A_{22}}= & \frac{1}{8}\left(3 A_{11}^{\prime}+A_{22}^{\prime}+4 A_{33}^{\prime}\right) b_{1} \\
- & \frac{1}{8}\left(3 A_{11}^{\prime}+A_{22}^{\prime}+4 A_{33}^{\prime}\right) b_{2} \\
+ & \frac{1}{8}\left(3 A_{11}^{\prime}-3 A_{22}^{\prime}\right) b_{3}-\frac{1}{8}\left(A_{11}^{\prime}-A_{22}^{\prime}\right) b_{4} \\
+ & \frac{1}{8}\left(A_{12}^{\prime}-A_{21}^{\prime}\right) b_{5}+\frac{1}{2}\left(A_{12}^{\prime}-A_{21}^{\prime}\right) b_{6}, \\
\overline{A_{23}}= & \bar{A}_{12}, \quad \bar{A}_{31}=\bar{A}_{12}, \quad \bar{A}_{33}=\bar{A}_{22},
\end{aligned}
$$

where

$$
\begin{aligned}
b_{1} & =\frac{\exp \left(\frac{1}{2} \pi \lambda\right)}{\exp \left(\frac{1}{2} \pi \lambda\right)-\lambda}, \quad b_{2}=\frac{\lambda}{\exp \left(\frac{1}{2} \pi \lambda\right)-\lambda}, \\
b_{3} & =\frac{\exp \left(\frac{1}{2} \pi \lambda\right)}{\exp \left(\frac{1}{2} \pi \lambda\right)-\lambda} \frac{1+\lambda^{2}}{9+\lambda^{2}}, \\
b_{4} & =\frac{\lambda}{\exp \left(\frac{1}{2} \pi \lambda\right)-\lambda} \frac{1+\lambda^{2}}{9+\lambda^{2}}, \\
b_{5} & =\frac{\exp \left(\frac{1}{2} \pi \lambda\right)}{\exp \left(\frac{1}{2} \pi \lambda\right)-\lambda} \frac{\lambda}{9+\lambda^{2}}, \\
b_{6} & =\frac{1}{\exp \left(\frac{1}{2} \pi \lambda\right)-\lambda} \frac{3+\lambda^{2}}{9+\lambda^{2}}, \\
b_{7} & =\frac{\left\{4 \exp \left(\frac{1}{2} \pi \lambda\right)-\left(4+\lambda^{2}\right)\right\}}{\left(\exp \left(\frac{1}{2} \pi \lambda\right)-\lambda\right) \lambda} \frac{1+\lambda^{2}}{4+\lambda^{2}},
\end{aligned}
$$




$$
\begin{aligned}
& b_{8}=\frac{\exp \left(\frac{1}{2} \pi \lambda\right)}{\exp \left(\frac{1}{2} \pi \lambda\right)-\lambda} \frac{1+\lambda^{2}}{4+\lambda^{2}}, \\
& b_{9}=\frac{1}{\exp \left(\frac{1}{2} \pi \lambda\right)-\lambda} \frac{1+\lambda^{2}}{4+\lambda^{2}}, \\
& b_{10}=\frac{\lambda}{\exp \left(\frac{1}{2} \pi \lambda\right)-\lambda} \frac{1+\lambda^{2}}{4+\lambda^{2}} .
\end{aligned}
$$

After we take the nonzero terms into Eq. (2.22) the effective thermal conductivity can be explicitly expressed as

$$
\begin{aligned}
K_{11}^{*}= & K_{\mathrm{m}}^{11}\left\{1+\left[0 . 2 5 f _ { 1 } \left[\left(b_{1}-b_{2}+3 b_{3}+b_{4}\right)\right.\right.\right. \\
& \times\left(S_{11}-1\right) X+\left(3 b_{1}-3 b_{2}-3 b_{3}-b_{4}\right) \\
& \left.\left.\times\left(S_{22}-1\right) Y\right]\right]\left[1+0.25 f_{1}\right. \\
& \times\left[\left(b_{1}-b_{2}+3 b_{3}+b_{4}\right) X\right. \\
& \left.\left.\left.+\left(3 b_{1}-3 b_{2}-3 b_{3}-b_{4}\right) Y\right]\right]^{-1}\right\}
\end{aligned}
$$

and

$$
\begin{aligned}
K_{22}^{*}= & K_{\mathrm{m}}^{22}\left\{1+\left[0 . 1 2 5 f _ { 1 } \left[\left(3 b_{1}-3 b_{2}-3 b_{3}-b_{4}\right)\right.\right.\right. \\
& \times\left(S_{11}-1\right) X+\left(5 b_{1}-5 b_{2}+3 b_{3}+b_{4}\right) \\
& \left.\left.\times\left(S_{22}-1\right) Y\right]\right]\left[1+0.125 f_{1}\right. \\
& \times\left[\left(3 b_{1}-3 b_{2}-3 b_{3}-b_{4}\right) X\right. \\
& \left.\left.\left.+\left(5 b_{1}-5 b_{2}+3 b_{3}+b_{4}\right) Y\right]\right]^{-1}\right\}
\end{aligned}
$$

where

$$
X=\frac{K_{\mathrm{f}}^{11}-K_{\mathrm{m}}^{11}}{K_{\mathrm{m}}^{11} S_{11}-K_{\mathrm{m}}^{11}-K_{\mathrm{f}}^{11} S_{11}}
$$

and

$$
Y=\frac{K_{\mathrm{f}}^{22}-K_{\mathrm{m}}^{22}}{K_{\mathrm{m}}^{22} S_{22}-K_{\mathrm{m}}^{22}-K_{\mathrm{f}}^{22} S_{22}}
$$

When $\lambda \rightarrow 0$ and $\lambda \rightarrow \infty, b_{1}, b_{2}, b_{3}, b_{4}, b_{5}, b_{6}, b_{7}$, $b_{8}, b_{9}$ and $b_{10}$ can be represented as

$$
\begin{array}{lll}
\lim _{\lambda \rightarrow 0} b_{1}=1, & \lim _{\lambda \rightarrow 0} b_{2}=0, & \lim _{\lambda \rightarrow 0} b_{3}=\frac{1}{9}, \\
\lim _{\lambda \rightarrow 0} b_{4}=0, & \lim _{\lambda \rightarrow 0} b_{5}=0, & \lim _{\lambda \rightarrow 0} b_{6}=\frac{1}{3}, \\
\lim _{\lambda \rightarrow 0} b_{7}=\frac{1}{2} \pi, & \lim _{\lambda \rightarrow 0} b_{8}=\frac{1}{4}, & \lim _{\lambda \rightarrow 0} b_{9}=\frac{1}{4},
\end{array}
$$

$$
\begin{array}{lll}
\lim _{\lambda \rightarrow 0} b_{10}=0, & \\
\lim _{\lambda \rightarrow \infty} b_{1}=1, & \lim _{\lambda \rightarrow \infty} b_{2}=0, & \lim _{\lambda \rightarrow \infty} b_{3}=1, \\
\lim _{\lambda \rightarrow \infty} b_{4}=0, & \lim _{\lambda \rightarrow \infty} b_{5}=0, & \lim _{\lambda \rightarrow \infty} b_{6}=0, \\
\lim _{\lambda \rightarrow \infty} b_{7}=0, & \lim _{\lambda \rightarrow \infty} b_{8}=1, & \lim _{\lambda \rightarrow \infty} b_{9}=0, \\
\lim _{\lambda \rightarrow \infty} b_{10}=0, & &
\end{array}
$$

when $\lambda \rightarrow 0$, substituting Eq. (4.5) into (4.3) leads to

$$
\begin{aligned}
& \bar{A}_{11}=\frac{1}{3}\left(A_{11}^{\prime}+2 A_{22}^{\prime}-A_{12}^{\prime}-A_{21}^{\prime}\right) \\
& \bar{A}_{22}=\bar{A}_{33}=\frac{1}{6}\left(2 A_{11}^{\prime}+A_{22}^{\prime}+3 A_{33}^{\prime}+A_{21}^{\prime}+A_{21}^{\prime}\right) .
\end{aligned}
$$

From Eq. (4.6) we know that as $\lambda \rightarrow 0$, it represents the short fibers are randomly distributed in the matrix. Therefore, $\bar{A}$ is an isotropic tensor.

When $\lambda \rightarrow \infty$, substituting (4.5) into (4.3) leads to

$\bar{A}=A^{\prime}$

From Eq. (4.7) we know that as $\lambda \rightarrow \infty$, it represents the short fibers are perfectly aligned in the matrix.

\subsubsection{Numerical results}

It is now of interest to see how the fiber distribution - (i) when it changes from uniaxial directional $(\lambda=\infty)$ to random distribution $(\lambda=0)$, (ii) the volume fraction, $f_{1}$, (iii) the aspect ratio, $l / d$, of the reinforced fibers - would affect the thermal conductivity of a transversely isotropic composite. To this end we used the properties of glass fibers and polyphenylene sulfide (PPS) matrix in our calculations. The thermal conductivities are

PPS: $K_{\mathrm{m}}^{11}=2 \mathrm{~mW} / \mathrm{cm} \cdot \mathrm{K}$

Glassfibers: $K_{\mathrm{f}}^{11}=10.4 \mathrm{~mW} / \mathrm{cm} \cdot \mathrm{K}$

In reading these results, we are reminded once again that direction 1 is the infinitely extended direction, direction 2 is perpendicular to the surface of the thickness, and direction 3 is along the width. Compared with the longitudinal thermal conductivities of $K_{\mathrm{m}}^{11}=2 \mathrm{~mW} / \mathrm{cm} \cdot \mathrm{K}$ for the PPS matrix, the thermal conductivity of the composites are significantly higher, clearly revealing the reinforcing effect of the 
fibers. To gain deeper understanding of the effects of various parameters, we have calculated and plotted the thermal conductivities of $f_{1}=30 \%$ glass-fiberreinforced PPS as functions of the aspect ratio, $l / d$, in Fig. 5a and b. They show that all the thermal conductivities for randomly distributed of glass-fiber reinforced composites ( $\lambda$ is small) increase with increasing $l / d$. The thermal conductivities $K_{11}$ increase with increasing $l / d$ for all $\lambda$. The effect becomes saturated above $l / d=50$. These results emphasize the importance of choosing suitable processing technique to increase the fiber aspect ratio, $l / d$.
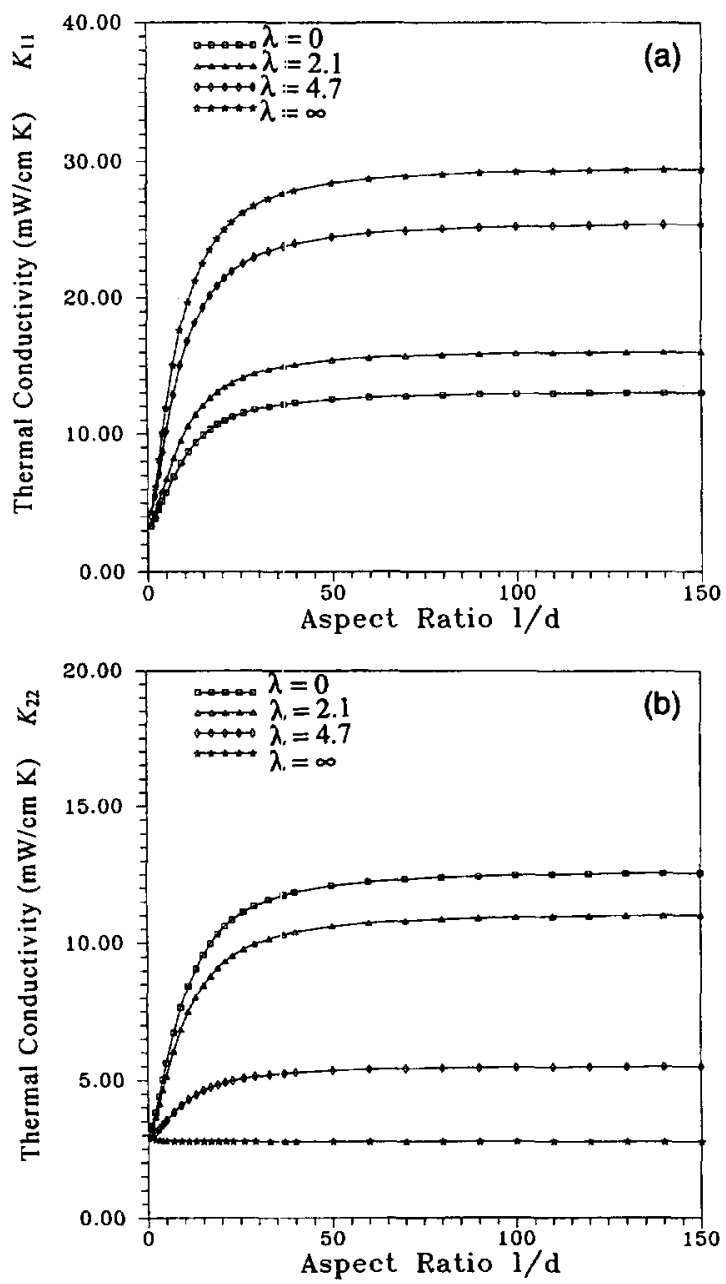

Fig. 5. The fiber orientation factor $\lambda$ dependence of the thermal conductivity as functions of aspect ratio, $l / d$, at $f_{1}=30 \%$; (a) $K_{11}$,(b) $K_{22}$.
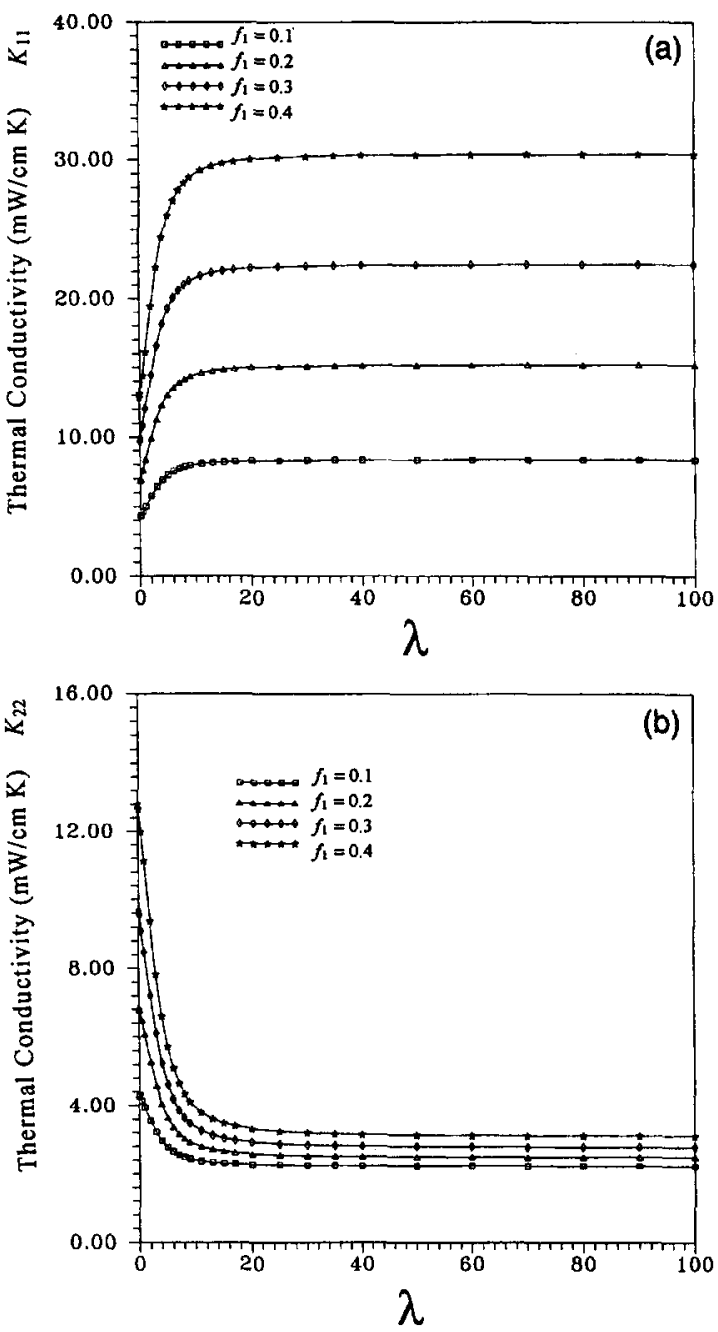

Fig. 6. The volume fractions dependence of the thermal conductivity as functions of fiber orientation factor, $\lambda$, at $l / d=15$; (a) $K_{11}$, (b) $K_{22}$.

Next we turn our attention to the fiber volume fraction, $f_{1}$, dependence of thermal conductivities as function of $\lambda$. The values of $f_{1}=0.1,0.2,0.3$ and 0.4 are chosen for demonstration. The thermal conductivities of a transversely isotropic composite as function of $\lambda$ are depicted in Fig. 6a and $b$. The thermal conductivities $K_{11}$ increases with increasing $\lambda$ but for $K_{22}$ decrease with increasing $\lambda$. They show that the more oriented ( $\lambda$ increase) the more reinforcing effect in 1-direction, but vice versus in the other direction. The effect becomes saturated above $\lambda=10$. 


\section{Conclusions}

The factors that influence the thermal conductivity of FRTP include the thermal conductivity of the fibers and the matrix, the volume fraction $f_{1}$, the aspect ratio $l / d$, and the orientation parameter $\lambda$, of the fiber. The two very important variables, $l / d$ and $\lambda$, can be controlled from the processing technique. A very special case is that the surface layer has the higher $\lambda$, and the middle layer has the lower one. As the experimental data observed, we can find that the parameter $\lambda$ can be obtained from the cumulative distribution $F(\theta)$. The aspect ratio $l / d$ can be decided before processing.

As we have known before, the effective thermal conductivity can be influenced by the thermal conductivity of the fiber much strongly than by the matrix. For this reason, if we want to enhance the properties of the composites, the choice of the fiber is very important. In this paper, we find that the carbon fiber is much better choice than glass fiber for higher thermal conductivity of the composites.

The basic idea in this paper comes from the Mori-Tanaka's mean field theory and the Eshelby's equivalent inclusion method. We also introduce Kacir's orientation distribution function $\rho(\theta)=$ $\lambda \mathrm{e}^{-\lambda \theta}$ in this paper to describe the fiber orientation. The theoretical models developed in this paper provide us a very simple, clear form to predict the thermal conductivity of the composites.

\section{Acknowledgements}

This work was supported by the National Science Council of R.O.C. under grant NSC-83-0401-E002145.

\section{Appendix A. Components of Eshelby's $S_{i j}$ tensor}

Let $t$ be the ratio of the inclusion $(=l / d)$ and $g$ be given by

$g=\frac{t}{\left(t^{2}-1\right)^{3 / 2}}\left[t\left(t^{2}-1\right)^{1 / 2}-\cosh ^{-1} t\right]$

for prolate inclusions

$g=\frac{t}{\left(1-t^{2}\right)^{3 / 2}}\left[\cos ^{-1} t-t\left(1-t^{2}\right)^{1 / 2}\right]$ for oblate inclusions

$g=2 / 3$ for spheres.

For a spherical inclusion aligned along 1-axis, the components of Eshelby's tensor $S_{i j}$ are given by

$S_{i j}=0$ if $i \neq j$.

For spherical inclusion they simplified to

$S_{11}=S_{22}=S_{33}=2 g=1 / 3$.

For prolate spheroid, one has

$S_{11}=1-g$

and

$S_{22}=S_{33}=g / 2$.

For oblate spheroid, one has

$S_{11}=1-g$

and

$S_{22}=S_{33}=g / 2$.

\section{References}

Chou, T.W. and S. Nomura, (1980-1981), Fiber orientation effects on the thermoelastic properties of short-fiber composites, Fibre Sci. Tech. 14, 279-291.

Choy, C.L., W.P. Leung, K.W. Kowk and O. Lau Felix (1992), Elastic moduli and thermal conductivity of injection-molded short-fiber-reinforced thermoplastics, Polym. Compos. 13, 69-80.

Eshelby, J.D. (1957), The determination of the elastic field of an ellipsoidal inclusion and related problems, Proc. R. Soc. London, A 241, 376-396.

Hatta, M. and M. Taya (1985), Effective thermal conductivity of a misoriented short fiber composite, J. Appl. Phys 58(7), 24782486.

Kacir, L., O. Ishai and M. Narkis (1978), Oriented short glass-fiber composites: IV. Dependence of mechanical properties on the distribution of fiber orientations, Polym. Eng. Sci. 18(1), 45-52.

Kacir, L., M. Narkis and O. Ishai, (1975), Oriented short glass-fiber composites: I. Preparation and statistical analysis of aligned fiber mats, Polym. Eng. Sci. 15, 525-531.

Kacir, L., M. Narkis and O. Ishai (1975), Oriented short glass-fiber composites: III. Structure and mechanical properties of molded sheets, Polym. Eng. Sci. 17, 234-241.

Mori, T. and K. Tanaka (1973), Average stress in matrix and average elastic energy of materials with misfitting inclusions, Acta Metall. 21, 571-574. 